\title{
A Riemannian rank-adaptive method for low-rank optimization ${ }^{\text {th }}$
}

\author{
Guifang Zhou ${ }^{\mathrm{a}}$, Wen Huang ${ }^{\mathrm{b}}$, Kyle A. Gallivan ${ }^{\mathrm{a}}$, Paul Van Dooren ${ }^{\mathrm{b}}$, \\ P.-A. Absil ${ }^{\mathrm{b}, *}$ \\ ${ }^{a}$ Department of Mathematics, Florida State University, 1017 Academic Way, Tallahassee, \\ FL 32306-4510, USA \\ ${ }^{b}$ ICTEAM Institute, Université catholique de Louvain, Avenue G. Lemaître 4, 1348 \\ Louvain-la-Neuve, Belgium
}

\begin{abstract}
This paper presents an algorithm that solves optimization problems on a matrix manifold $\mathcal{M} \subseteq \mathbb{R}^{m \times n}$ with an additional rank inequality constraint. The algorithm resorts to well-known Riemannian optimization schemes on fixed-rank manifolds, combined with new mechanisms to increase or decrease the rank. The convergence of the algorithm is analyzed and a weighted low-rank approximation problem is used to illustrate the efficiency and effectiveness of the algorithm.
\end{abstract}

Keywords: low-rank optimization, rank-constrained optimization, Riemannian manifold, fixed-rank manifold, low-rank approximation 2010 MSC: 65K05, 90C30, 15A03

\section{Introduction}

We consider low-rank optimization problems of the following form:

$$
\min _{X \in \mathcal{M}_{\leq k}} f(X)
$$

where $\mathcal{M}$ is a submanifold of $\mathbb{R}^{m \times n}$,

$$
\mathcal{M}_{\leq k}:=\{X \in \mathcal{M} \mid \operatorname{rank}(X) \leq k\}
$$

with $k \leq \min (m, n)$, and $f$ is a real-valued function on $\mathcal{M}_{\leq k}$. The notation

$$
\mathcal{M}_{r}:=\{X \in \mathcal{M} \mid \operatorname{rank}(X)=r\}
$$

\footnotetext{
This paper presents research results of the Belgian Network DYSCO (Dynamical Systems, Control, and Optimization), funded by the Interuniversity Attraction Poles Programme initiated by the Belgian Science Policy Office. This work was supported by the National Science Foundation under grant NSF-1262476 and by FNRS under grant PDR T.0173.13.

* Corresponding author
} 
will also be used frequently. Typical choices for $\mathcal{M}$ are $\mathbb{R}^{m \times n}$ itself and the Frobenius sphere, i.e., the set of all $m \times n$ matrices of fixed Frobenius norm.

Applications of (1) appear notably in machine learning, e.g., for collaborative

5 filtering [1, 2], multi-class classification [3], multi-response regression [4, 5], learning a function over pairs of points [6], and learning a low-rank similarity measure [7]. Applications of low-rank optimization are also found in other areas such as systems and control [8, 9] and computer vision [10, 11].

An increasingly popular way to approach problem 1 is to consider the re10 lated but simpler problem $\min _{X \in \mathbb{R}_{k}^{m \times n}} f(X)$, where $\mathbb{R}_{k}^{m \times n}=\left\{X \in \mathbb{R}^{m \times n}\right.$ | $\operatorname{rank}(X)=k\}$ in view of the notation (2); see, e.g., 12, 13, 7, 14. Since $\mathbb{R}_{k}^{m \times n}$ is a submanifold of $\mathbb{R}^{m \times n}$ of dimension $(m+n-k) k$ (see [15, Ch. 5, Prop. 1.14]), this simpler problem can be solved using Riemannian optimization techniques such as those presented in [16, 17, 18, 19, 20. However, a disadvantage is that

15 the manifold $\mathbb{R}_{k}^{m \times n}$ is not closed in $\mathbb{R}^{m \times n}$, which jeopardizes the well-posedness of the optimization problem and complicates the convergence analysis of optimization methods if the iterates cannot be assumed to stay safely away from $\mathbb{R}_{\leq k-1}^{m \times n}$.

Very recently a more global view of a projected line-search method on $\mathbb{R}_{\leq k}^{m \times n}=\left\{X \in \mathbb{R}^{m \times n} \mid \operatorname{rank}(X) \leq k\right\}$ along with a convergence analysis has been developed in [21]. In [22], the results of [21] have been exploited to propose an algorithm that successively increases the rank by a given constant. Its convergence to critical points can be deduced from [21, Th. 3.9]; it relies on the assumption, often satisfied in practice, that the limit points have rank $k$.

25 Under this assumption, a line-search method on $\mathbb{R}_{\leq k}^{m \times n}$ is ultimately the same as a line-search method on $\mathbb{R}_{k}^{m \times n}$.

In this paper, we develop a Riemannian rank-adaptive algorithm for the optimization problem (1). Its main features are as follows. First, the feasible set $\mathcal{M}_{\leq k}$ is more general than the set $\mathbb{R}_{\leq k}^{m \times n}$ considered in [21, 22]. Second, 30 the proposed algorithm increases or decreases the rank by an adaptively-chosen amount as the iteration proceeds. The rank update mechanism is governed by parameters that the user can adjust to strike a balance between the goals of (i) saving on space and time complexity by reducing the rank and (ii) achieving higher accuracy by increasing the rank. Finally, theoretical convergence 35 results are given, and the proposed method is shown on numerical experiments to outperform state-of-the-art methods on a weighted low-rank approximation problem.

The rest of this paper is organized as follows. Standing assumptions are gathered in the next section. The proposed method is presented in Section 3 and 40 analyzed in Section 4. Implementation practicalities are discussed in Section 5 Numerical experiments are reported in Section 6, and conclusions are drawn in Section 7 .

A preliminary version of this work can be found in $[23]$. 


\section{Notation, Definitions, and Standing Assumptions}

The notation $\mathcal{M}_{r}$ and $\mathcal{M}_{\leq r}$ defined above will be used frequently. The notation $f_{\mathrm{F}}$ stands for an extension of $f$ on $\mathcal{M}$ (see Assumption 3 below) and $f_{r}$ denotes the restriction of $f$ to $\mathcal{M}_{r}$.

Throughout the paper, the following assumptions are in force.

Assumption 1. $\overline{\mathcal{M}_{r}} \subseteq \mathcal{M}_{\leq r}$ for all positive integers $r \leq k$, where $\overline{\mathcal{M}_{r}}$ stands 50 for the closure of $\mathcal{M}_{r}$.

Observe that, since the closure of an intersection is a subset of the intersection of the closures and since $\overline{\mathbb{R}_{r}^{m \times n}}=\mathbb{R}_{<r}^{m \times n}$, it follows that the above assumption holds whenever the submanifold $\mathcal{M}$ is a closed subset of $\mathbb{R}^{m \times n}$. It is useful to bear in mind that a sequence of rank-r matrices can converge to a lower-rank matrix but not to a larger-rank matrix.

The next assumption is crucial to the Riemannian aspect of the proposed Riemannian rank-adaptive method:

Assumption 2. $\mathcal{M}_{r}$ is a submanifold of $\mathbb{R}^{m \times n}$, for all positive integers $r \leq k$.

We need the cost function $f$ to be sufficiently smooth for gradient-descent techniques to be applicable:

Assumption 3. The cost function $f$ admits a continuously differentiable extension $f_{\mathrm{F}}$ on a neighborhood of $\mathcal{M}_{\leq k}$ in $\mathcal{M}$.

The reader will observe that neither the size of the neighborhood nor the choice of the extension will have an impact on the proposed method.

The tangent cone to a set $S \subseteq \mathbb{R}^{m \times n}$ at $X \in \mathbb{R}^{m \times n}$ is the set

$$
\mathrm{T}_{X} S:=\left\{\dot{\gamma}(0) \mid \gamma \in C^{1}, \gamma(0)=X, \exists \delta>0: \forall t \in(0, \delta): \gamma(t) \in S\right\},
$$

where $\dot{\gamma}(0)$ denotes the derivative of curve $\gamma$ at 0 . This definition of $\mathrm{T}_{X} S$ is motivated by the goal of conducting line searches along smooth (i.e., $C^{1}$ ) curves. Observe that $\mathrm{T}_{X} S=\emptyset$ when $X \notin \bar{S}$.

We point out that, for any $X \in \mathcal{M}_{r}$, the tangent cones are nested as follows: $\mathrm{T}_{X} \mathcal{M}_{\leq 0} \subseteq \mathrm{T}_{X} \mathcal{M}_{\leq 1} \subseteq \cdots \subseteq \mathrm{T}_{X} \mathcal{M}$. The tangent cones $\mathrm{T}_{X} \mathcal{M}_{\leq r}$ and $\mathrm{T}_{X} \mathcal{M}$

70 are actually linear spaces since $\mathcal{M}$ and $\mathcal{M}_{r}$ are manifolds and $\mathcal{M}_{\leq r}$ is identical to $\mathcal{M}_{r}$ locally around $X \in \mathcal{M}_{r}$. Moreover, we have $\mathrm{T}_{X} \mathcal{M}_{\leq s}=\emptyset$ for all $s<r$. This justifies the following definition.

Definition 1 (update-rank). Let $X \in \mathcal{M}$ and $\eta_{X} \in \mathrm{T}_{X} \mathcal{M}$. The update-rank of $\eta_{X}$ is the unique integer $r$ such that $\eta_{X} \in \mathrm{T}_{X} \mathcal{M}_{\leq r} \backslash \mathrm{T}_{X} \mathcal{M}_{\leq r-1}$, with $A \backslash B$ denoting the set difference $\{x \in A \mid x \notin B\}$.

For the purpose of conducting line searches along given directions while keeping the rank under control, we will need $\mathcal{M}$ to be endowed with a curveselection mechanism defined as follows, where $\mathrm{T} \mathcal{M}:=\bigsqcup_{X \in \mathcal{M}} \mathrm{T}_{X} \mathcal{M}$ denotes the tangent bundle of $\mathcal{M}$. 
Definition 2 (Rank-related retraction). In the context of problem (1), a mapping $\tilde{R}: \mathrm{T} \mathcal{M} \rightarrow \mathcal{M}$ is a rank-related retraction if, for all $X_{*} \in \mathcal{M}_{\leq k}$, there exists $\delta_{X_{*}}>0$ and a neighborhood $\mathcal{U}$ of $X_{*}$ in $\mathcal{M}_{\leq k}$ such that, for all $X \in \mathcal{U}$ and all $\xi_{X} \in \mathrm{T}_{X} \mathcal{M}_{\leq k}$ with $\left\|\xi_{X}\right\|=1$, it holds that (i) $\tilde{R}_{X}(0)=X$, where $\tilde{R}_{X}$ denotes the restriction of $\tilde{R}$ to $\mathrm{T}_{X} \mathcal{M}$ and 0 stands for the zero vector in $\mathrm{T}_{X} \mathcal{M}$, (ii) $\left[0, \delta_{X_{*}}\right) \ni t \mapsto \tilde{R}_{X}\left(t \xi_{X}\right)$ is smooth and $\tilde{R}_{X}\left(t \xi_{X}\right) \in \mathcal{M}_{\leq \tilde{r}}$ for all $t \in\left[0, \delta_{X_{*}}\right)$, where $\tilde{r}$ is the update-rank of $\xi_{X}$, (iii) $\left.\frac{d}{d t} \tilde{R}_{X}\left(t \xi_{X}\right)\right|_{t=0}=\xi_{X}$.

Note that $\tilde{R}_{X}$ is not necessarily a retraction on $\mathcal{M}$ in the sense given in [24, [16], since it may not be smooth on the tangent bundle TM. A specific rankrelated retraction is given in Section 5

$90 \quad$ Observe that in point (ii) of Definition 2, we require $\tilde{R}_{X}\left(t \xi_{X}\right)$ to belong to $\mathcal{M}_{\leq \tilde{r}}$ but not necessarily to $\mathcal{M}_{\tilde{r}}$. Indeed we found that the condition $\tilde{R}_{X}\left(t \xi_{X}\right) \in$ $\mathcal{M}_{\tilde{r}}$ would be cumbersome to enforce while being unnecessary for the convergence analysis.

We let grad $f_{\mathrm{F}}(X)$ denote the Riemannian gradient of $f_{\mathrm{F}}$ at $X \in \mathcal{M}$. It can be obtained by considering any smooth extension of $f_{\mathrm{F}}$ around $X$ in $\mathbb{R}^{m \times n}$ and taking the projection to $\mathrm{T}_{X} \mathcal{M}$ of its Euclidean gradient at $X$; see [16, (3.37)]. Likewise, $\operatorname{grad} f_{r}(X)$ denotes the Riemannian gradient of $f_{r}$ at $X \in \mathcal{M}_{r}$, and it is obtained by projecting grad $f_{\mathrm{F}}(X)$ onto the tangent space $\mathrm{T}_{X} \mathcal{M}_{r}$.

Throughout the paper, $\|\cdot\|$ denotes the Frobenius norm and $\langle\cdot, \cdot\rangle$ the Frobenius inner product.

Consider $X \in \mathcal{M}, \xi \in \mathrm{T}_{X} \mathcal{M}$, and a positive integer $r$. The set of best approximations of $\xi$ in $\mathrm{T}_{X} \mathcal{M}_{\leq r}$ is denoted by $\mathrm{P}_{\mathrm{T}_{X} \mathcal{M}_{\leq r}}(\xi)$. Note that this set may contain more than one point. (In the case where $\mathcal{M}=\mathbb{R}^{m \times n}$, this follows directly from $(12)$ and the non-uniqueness of a best low-rank approximation.) However, as indicated in 21, §2.1] (or see Lemma 1 below), all its elements have the same norm, hence $\left\|\mathrm{P}_{\mathrm{T}_{X} \mathcal{M}_{\leq r}}(\xi)\right\|$ is well defined. We say that $X$ is a critical point of $f$ if $\left\|\mathrm{P}_{\mathrm{T}_{X} \mathcal{M}_{\leq k}}\left(\operatorname{grad} f_{\mathrm{F}}(X)\right)\right\|=0$. (It can be seen that this notion does not depend on the chosen extension $f_{\mathrm{F}}$ of $f$.)

\section{A Riemannian Rank-Adaptive Algorithm}

The proposed method is listed in Algorithm 3, but we invite the reader to first read the more reader-friendly description in Section 3.1 and to refer to the pseudocode in Algorithm 3 when needed.

\subsection{Algorithm description}

We first discuss the two subprograms, Algorithms 1 and 2 , called by Algorithm 3. 


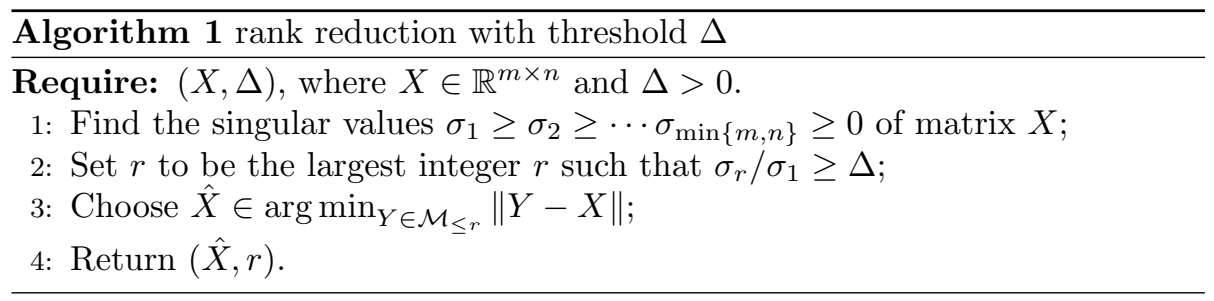

The output $\hat{X}$ of Algorithm 1 is a best approximation of $X$ in $\mathcal{M}_{\leq r}$, where $r$ is the number (counting multiplicities) of singular values of $X$ that are larger than $\sigma_{1} \Delta$, with $\sigma_{1}$ the largest singular value of $X$. Observe that $\hat{X}$ is simply $X$ in the plausible case where $X$ is already in $\mathcal{M}_{\leq r}$. In the case $\mathcal{M}=\mathbb{R}^{m \times n}$, ${ }_{120}$ Algorithm 1 consists in setting to zero the singular values of $X$ that are smaller than $\sigma_{1} \Delta$.

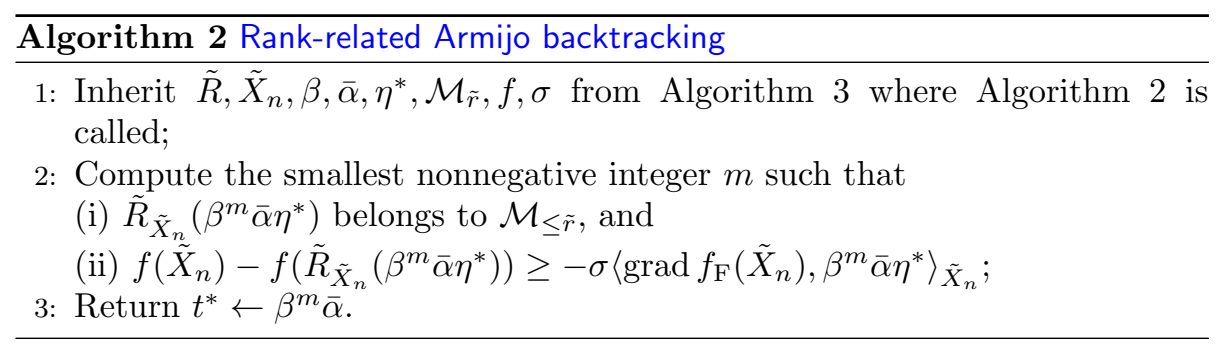

Algorithm 2 differs from the Riemannian Armijo backtracking [16, Definition 4.2.2] by the safeguard (i). This safeguard is used because, in view of its $\delta_{X_{*}}$, Definition 2 does not ensure that $\tilde{R}_{\tilde{X}_{n}}\left(\beta^{m} \bar{\alpha} \eta^{*}\right)$ is in $\mathcal{M}_{\leq \tilde{r}}$ unless $m$ is sufficiently large.

Let us now turn to the main part (Algorithm 3) of the proposed Riemannian rank-adaptive method. The underlying idea is to alternate between the following two tasks.

1. Riemannian update (line 3 of Algorithm 3): given an iterate $X_{n}$ in $\mathcal{M}_{r}$ with $r \leq k$, run a Riemannian optimization method on the manifold $\mathcal{M}_{r}$, which returns a point $\tilde{X}_{n}$ in $\mathcal{M}_{r}$ when a suitably chosen stopping criterion is satisfied.

2. Rank-related update: generate a new iterate $X_{n+1}$ in $\mathcal{M}_{\leq k}$ (line 10, line 13 , or line 21), update the tolerances in the suitably chosen stopping criterion (line 11, line 13, line 18), increment $n$, and return to the Riemannian update.

We assume that the Riemannian optimization method invoked in the Riemannian update (line 3) is a descent iteration enjoying a global convergence property shared by all respectable such algorithms:

140 Assumption 4 (globally convergent Riemannian optimization method). Let $\left\{Z_{j}\right\}$ denote an infinite sequence generated by the Riemannian optimization 


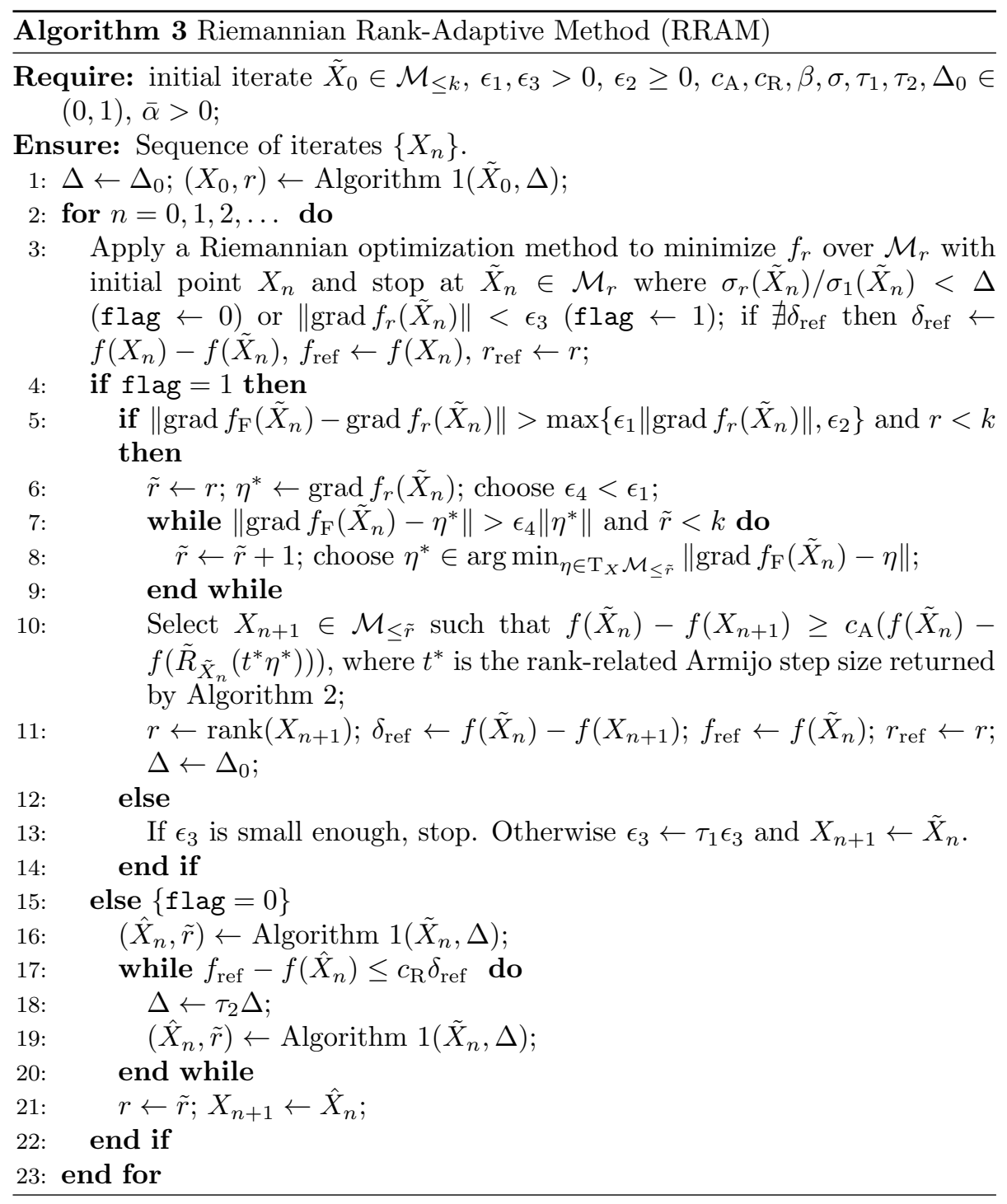

method of line 3 of Algorithm 3. Then $f\left(Z_{j+1}\right) \leq f\left(Z_{j}\right)$ for all $j$. If $Z_{*}$ is a limit point of $\left\{Z_{j}\right\}$ in $\mathcal{M}_{r}$ (i.e., if there is no drop of rank at the limit point), then $\operatorname{grad} f_{r}\left(Z_{*}\right)=0$.

145 Well-understood general-purpose Riemannian optimization methods abound that, when applied to $\mathcal{M}_{r}$, satisfy Assumption 4, see, e.g., [17, 18, 20, 19] for recent points of entry to the literature. Several suitable implementations on $\mathcal{M}_{r}$ 
are available in Matlab [25] and $\mathrm{C}++1$

The challenge is thus to suitably choose the stopping criterion of the Riemannian update and to handle the rank-related updates so as to achieve the features mentioned in the introduction.

The stopping criterion for the Riemannian update is given in line 3 of $\mathrm{Al}-$ gorithm 3. The Riemannian update returns with $f l a g=0$ if $\tilde{X}_{n}$ is found to be dangerously close to the lower-rank set $\mathcal{M}_{\leq r-1}$; otherwise it returns with $155 \quad \mathrm{flag}=1$ when $\left\|\operatorname{grad} f_{r}\left(\tilde{X}_{n}\right)\right\|$ becomes sufficiently small. (The "or" in line 3 is thus a "short-circuit or".) The danger announced by $f l a g=0$ comes from Assumption 4, which offers no guarantee on the limit points of the Riemannian update that have rank lower than $r$. Hence, when the iterates of the Riemannian optimization method come too close to $\mathcal{M}_{\leq r-1}$, one needs to take action. The $f l a g=0$ branch of the decision tree will be discussed in a moment.

Let us for now consider the case $\mathrm{flag}=1$, i.e., the Riemannian update (line 3 has returned $\tilde{X}_{n} \in \mathcal{M}_{r}$ sufficiently far away from $\mathcal{M}_{\leq r-1}$ and satisfying $\left\|\operatorname{grad} f_{r}\left(\tilde{X}_{n}\right)\right\|<\epsilon_{3}$. This indicates that $\tilde{X}_{n}$ is an approximate critical point of $f$ restricted to $\mathcal{M}_{r}$. However, it may still be possible to considerably reduce the value of $f$ if we let the rank of the iterate get larger than $r$. We thus resort to readily available information - namely the first-order information provided by $\operatorname{grad} f_{\mathrm{F}}\left(\tilde{X}_{n}\right)$ and grad $f_{r}\left(\tilde{X}_{n}\right)$, the latter being the projection of the former onto the tangent space $\mathrm{T}_{\tilde{X}_{n}} \mathcal{M}_{r}$ - to decide if a rank increase looks promising (line 5). Specifically, we check if the angle between $\operatorname{grad} f_{\mathrm{F}}\left(\tilde{X}_{n}\right)$ and $\operatorname{grad} f_{r}\left(\tilde{X}_{n}\right)$ is large, as measured by the condition

$$
\tan \left(\angle\left(\operatorname{grad} f_{\mathrm{F}}\left(\tilde{X}_{n}\right), \operatorname{grad} f_{r}\left(\tilde{X}_{n}\right)\right)\right)>\epsilon_{1},
$$

and if moreover their difference is large, as measured by the condition

$$
\left\|\operatorname{grad} f_{\mathrm{F}}\left(\tilde{X}_{n}\right)-\operatorname{grad} f_{r}\left(\tilde{X}_{n}\right)\right\|>\epsilon_{2} .
$$

The conditions are illustrated in Figure 1. If both these conditions are satisfied, and moreover the maximal rank $k$ is not reached (i.e., $r<k$ ), then we decide that we are in a favorable situation to attempt a rank increase. We now explore this branch of the decision tree.

165 The next issue is to decide by how much we allow the rank to increase. To this end, we increment $\tilde{r}$ from $r$ to at most $k$ until the tangent of the angle between $\operatorname{grad} f_{\mathrm{F}}\left(\tilde{X}_{n}\right)$ and its projection $\eta^{*}$ onto $\mathrm{T}_{X} \mathcal{M}_{\leq}$is smaller than some $\epsilon_{4}$ chosen smaller than $\epsilon_{1}$ (lines 6 9). At the end of this procedure, assuming that $\tilde{r}$ has not reached $k$, we can conclude that the update-rank of $\eta^{*}$ (Definition 1) 170 is $\tilde{r}>r$. Note that if $\mathcal{M}=\mathbb{R}^{m \times n}$ and $\tilde{r}=k$, then the choice of $\eta^{*}$ (line 8 is equivalent to the definition in [21, Corollary 3.3].

We then perform an update along $\eta^{*}$ by means of the rank-related retraction (Definition 2) that $\mathcal{M}$ needs to be endowed with, and we choose the step size by

$1 \sqrt{\text { http: //www . math.fsu.edu/ whuang2/ROPTLIB.htm }}$ 
an Armijo-type backtracking procedure (line 10). This yields the next iterate, $X_{n+1}$, whose rank is less than or equal to $\tilde{r}$ but not necessarily equal to $\tilde{r}$.

Note that this Armijo procedure (line 10 is the only place in the proposed method where a rank increase can possibly occur. Choosing $\epsilon_{1}$ and $\epsilon_{2}$ small makes the algorithm more prone to executing the Armijo step, hence to increasing the rank. Choosing $\epsilon_{2}>0$ may result in blocking the rank at a small value 180 for which the optimization problem (1) does not admit critical points, but the forthcoming Theorem 3 gives an upper bound on the "lack of criticality" of the output of the algorithm.

We now discuss the other branches of the decision tree. Still assume that $\mathrm{flag}=1$ but that we decide that we are not in a favorable situation to attempt a rank increase. Then we set $X_{n+1}$ to $\tilde{X}_{n}$ and we return to the Riemannian step, now with a more stringent tolerance $\epsilon_{3}$ (line 13 ).

Now consider the case $f l a g=0$, where we know that we need to take action because the Riemannian update over $\mathcal{M}_{r}$ has returned $\tilde{X}_{n}$ dangerously close to the lower-rank set $\mathcal{M}_{\leq r-1}$. The principle of the action is to perform a rank reduction by setting $X_{n+1}$ as the projection of $\tilde{X}_{n}$ to $\mathcal{M}_{<\tilde{r}}$, where $\tilde{r}$ is the $\Delta$-numerical rank of $\tilde{X}_{n}$ returned by Algorithm $1\left(\tilde{X}_{n}, \Delta\right)$. However, since the forthcoming convergence analysis relies crucially on the effect of the Armijo steps, we keep decreasing $\Delta$ (hence making the rank reduction less drastic) until the decrease of $f$ achieved by the latest Armijo step (or by the initial 195 Riemannian update if no Armijo step has been performed yet) is not too much unraveled by the rank reduction. The details are spelled out in line 16 and beyond.

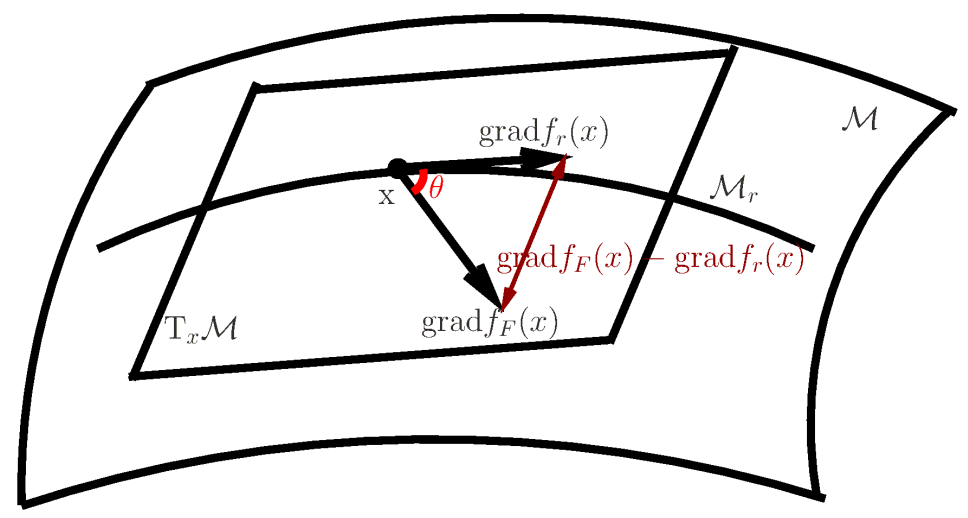

Figure 1: Illustration of conditions $(3)$ and (4). $\theta$ is the angle between $\operatorname{grad} f_{\mathrm{F}}(X)$ and $\operatorname{grad} f_{r}(X)$ and the length of the red arrow represents $\left\|\operatorname{grad} f_{\mathrm{F}}(X)-\operatorname{grad} f_{r}(X)\right\|$.

\subsection{Termination analysis}

Algorithm 3 is meant to generate an infinite sequence $\left\{X_{n}\right\}_{n=0,1, \ldots}$, whose asymptotic behavior is analyzed in Section 4 . In practice, a termination criterion 
can be based on the norm of the update vector $\eta^{*}$ and on various contextdependent considerations. Our purpose in this section is to make sure that all the steps of Algorithm 3 are well defined and terminate.

In view of Assumption 4 the Riemannian update in line 3 is guaranteed to terminate if the following assumption holds:

Assumption 5. The sublevel set $\left\{X \in \mathcal{M}_{\leq k}: f(X) \leq f\left(X_{0}\right)\right\}$ is compact.

Indeed, in view of Assumption 1 and the Bolzano-Weierstrass theorem, the sequence of iterates generated by the Riemannian optimization method has then at least one limit point, which is in $\mathcal{M}_{\leq r}$. Either this limit point is in $\mathcal{M}_{r}$ and thus, by Assumption 4 4 grad $f_{r}$ gets arbitrarily small; or this limit point is in $\mathcal{M}_{\leq r-1}$ and the Riemannian optimization method returns with $\mathrm{flag}=0$.

The while loop starting in line 7 obviously terminates in view of the condition $\tilde{r}<k$.

The Armijo backtracking procedure (Algorithm 2 called in line 10 terminates (i.e., the smallest nonnegative integer $m$ exists) in view of a classical argument since the function $\mathbb{R} \ni t \mapsto f\left(\tilde{R}_{\tilde{X}_{n}}\left(t \eta^{*}\right)\right) \in \mathbb{R}$ is differentiable around $t=0$, a consequence of Assumption 3 and Definition 2 .

In line 11, the fact that the rank may be numerically uncertain after the Armijo step is not an issue: if this is the case, then the next Riemannian update (line 3) will return immediately with $f l a g=0$ and a rank decrease will take place.

The while loop in line 17 is guaranteed to terminate: if it keeps being executed, then eventually no truncation occurs (i.e., $\hat{X}_{n}=\tilde{X}_{n}$ ), implying $f_{\text {ref }}-f\left(\hat{X}_{n}\right)=f_{\text {ref }}-f\left(\tilde{X}_{n}\right)>c_{\mathrm{R}} \delta_{\text {ref }}$.

\section{Convergence Analysis}

We now proceed to the convergence analysis of Algorithm 3 under the standing assumptions stated in Section 2 .

In Section 4.1 we consider the specific case where $\epsilon_{2}$ is set to zero, then in Section 4.2 we exploit our findings to handle the general case $\epsilon_{2} \geq 0$. Observe 230 that $\epsilon_{2}$ appears only in line 5 of Algorithm 3 . When it is set to zero, the first condition in line 5 - which must be satisfied to execute Armijo (line 10 -reduces to the angle condition (3). The motivation behind a choice of $\epsilon_{2}>0$ is that it makes the algorithm less inclined to execute Armijo, hence more inclined to keep the rank low, thus gaining in spatial complexity. The findings of our convergence 235 analysis indicate that the price to pay is the residual error that may subsist, in the sense that $\lim \inf _{n \rightarrow \infty}\left\|\mathrm{P}_{\mathrm{T}_{X_{n}} \mathcal{M}_{\leq k}}\left(\operatorname{grad} f_{\mathrm{F}}\left(X_{n}\right)\right)\right\|$ may not be zero; however, the residual error remains under control due to an upper bound proportional to $\epsilon_{2}$ guaranteed by Theorem 3 .

We need some preliminary work before stating and proving the convergence 240 results. Lemma 1 concerns the vector $\eta^{*}$ obtained in line 8 of Algorithm 3 . The first claim of Lemma 1 will be invoked in the proof of Theorem 2, while its second claim is an easy result that confirms a property announced in Section 2 
Lemma 1. Let $X \in \mathcal{M}_{r}$ and $\tilde{r} \geq r$. If $\eta^{*} \in \arg \min _{\eta \in \mathrm{T}_{X}} \mathcal{M}_{\leq \tilde{r}}\left\|\operatorname{grad} f_{\mathrm{F}}\left(\tilde{X}_{n}\right)-\eta\right\|$ then

$$
\left\langle\eta^{*}, \operatorname{grad} f_{\mathrm{F}}(X)\right\rangle=\left\|\eta^{*}\right\|^{2} .
$$

We also have $\left\|\eta^{*}\right\|^{2}=\left\|\operatorname{grad} f_{\mathrm{F}}(X)\right\|^{2}-\min _{\eta \in \mathrm{T}_{X}} \mathcal{M}_{\leq \tilde{r}}\left\|\operatorname{grad} f_{\mathrm{F}}\left(\tilde{X}_{n}\right)-\eta\right\|^{2}$.

Proof. Let $\eta^{*} \in \arg \min _{\eta \in \mathrm{T}_{X} \mathcal{M}_{<\tilde{r}}}\left\|\operatorname{grad} f_{\mathrm{F}}\left(\tilde{X}_{n}\right)-\eta\right\|$. Since the tangent cone $\mathrm{T}_{X} \mathcal{M}_{\leq \tilde{r}}$ is indeed a cone, $t \eta^{*} \in \mathrm{T}_{X} \mathcal{M}_{\leq \tilde{r}}$ for all $t>0$. Therefore

$$
\left.\frac{d}{d t}\left\|\operatorname{grad} f_{\mathrm{F}}(X)-t \eta^{*}\right\|^{2}\right|_{t=1}=0
$$

which implies

$$
\left\langle\eta^{*}, \operatorname{grad} f_{\mathrm{F}}(X)\right\rangle=\left\langle\eta^{*}, \eta^{*}\right\rangle=\left\|\eta^{*}\right\|^{2},
$$

which is the first claim. We thus have the orthogonality condition $\left\langle\eta^{*}, \operatorname{grad} f_{\mathrm{F}}(X)-\right.$ $\left.{ }_{245} \eta^{*}\right\rangle=0$, from which it follows that $\left\|\operatorname{grad} f_{\mathrm{F}}(X)\right\|^{2}=\left\|\operatorname{grad} f_{\mathrm{F}}(X)-\eta^{*}\right\|^{2}+\left\|\eta^{*}\right\|^{2}$, yielding the second claim.

The analysis of the Armijo step makes use of the following assumption, which is satisfied in particular when the rank-related retraction $\tilde{R}$ is the one proposed in Section 5 ,

Assumption 6 (locally radially L-C ${ }^{1}$ ). The lifted function

$$
\hat{f}: \mathrm{TM} \rightarrow \mathbb{R}: \xi \mapsto f \circ \tilde{R}(\xi) .
$$

is locally radially Lipschitz continuously differentiable (locally radially L- $C^{1}$ ), that is, for all $X_{*} \in \mathcal{M}_{\leq k}$, there exists $\beta_{R L}>0, \delta_{R L}>0$, and a neighborhood $\mathcal{U}$ of $X_{*}$ in $\mathcal{M}_{\leq k}$ such that, for all $X \in \mathcal{U}$, for all $\xi \in \mathrm{T}_{X} \mathcal{M}_{\leq k}$ with $\|\xi\|=1$, and for all $t<\bar{\delta}_{R L}$, it holds that

$$
\left|\frac{d}{d \tau} \hat{f}_{X}(\tau \xi)\right|_{\tau=t}-\left.\frac{d}{d \tau} \hat{f}_{X}(\tau \xi)\right|_{\tau=0} \mid \leq \beta_{R L} t
$$

\subsection{Convergence Analysis with $\epsilon_{2}=0$}

The main global convergence properties of Algorithm 3 for $\epsilon_{2}=0$ are stated in Theorem 2 below. In a nutshell, it shows that the best approximation of $\operatorname{grad} f_{\mathrm{F}}\left(X_{n}\right)$ in $\mathrm{T}_{X_{n}} \mathcal{M}_{\leq k}$ gets arbitrarily small for some $n$ large enough. Its proof can be viewed as an extension to the rank-adaptive setting of the well-

255 known fact that an occasional steepest descent step is sufficient to guarantee global convergence in a Euclidean setting [26, p. 41] and more generally in the Riemannian setting [27. As we will see, however, spelling out the proof details is not straightforward.

Theorem 2. Under the standing assumptions (Section 2) and Assumptions 4 ${ }_{260}$ 6. let $\left\{X_{n}\right\}$ be an infinite sequence of iterates generated by Algorithm 3 with $\epsilon_{2}=0$. Then $\liminf \operatorname{in}_{n \rightarrow \infty}\left\|\mathrm{P}_{\mathrm{T}_{X_{n}} \mathcal{M}_{\leq k}}\left(\operatorname{grad} f_{\mathrm{F}}\left(X_{n}\right)\right)\right\|=0$. 
Proof. We will distinguish two cases.

Case 1: Armijo (line 10) is executed infinitely many times. In this case, the claim follows from a fairly standard Armijo-type analysis invoking Assumption 6. Details are given below.
Case 2: Armijo (line 10 is executed finitely many times. Since the Armijo step is the only step of Algorithm 3 where the rank can increase, it follows that $X_{n}$ stays in a fixed-rank manifold $\mathcal{M}_{r}$ for all $n$ large enough. The rank reduction mechanism of Algorithm 3 does not allow the iterates to approach $\mathcal{M}_{\leq r-1}$ (see details below) and the stronger claim $\liminf _{n \rightarrow \infty}\left\|\operatorname{grad} f\left(X_{n}\right)\right\|=0$ (when $r=k$ ) or even $\liminf { }_{n \rightarrow \infty}\left\|\operatorname{grad} f_{\mathrm{F}}\left(X_{n}\right)\right\|=0$ (when $r<k$ ) follows from Assumption 4 .

Case 1 (details): Since Armijo (line 10 is executed infinitely many times and the sequence of iterates is bounded (a consequence of Assumption 5), it follows that there exists a convergent subsequence $\left\{X_{n_{j}}\right\}$ of iterates at which Armijo is executed. Let $X_{*} \in \mathcal{M}_{\leq k}$ denote the limit of the subsequence. In view of line 10 of Algorithm 3 .

$$
f\left(X_{n_{j}}\right)-f\left(X_{n_{j}+1}\right) \geq-c_{\mathrm{A}} \sigma \alpha_{n_{j}}\left\langle\operatorname{grad} f_{\mathrm{F}}\left(X_{n_{j}}\right), \eta_{n_{j}}^{*}\right\rangle_{X_{n_{j}}},
$$

where $\sigma \in(0,1)$ is a parameter of Algorithm 3 and $\eta_{n_{j}}^{*}$, resp. $\alpha_{n_{j}}$, denotes the $\eta^{*}$, resp. $t^{*}$, produced by line 10 at iteration $n_{j}$. Note that Algorithm 3 is not a descent iteration (the value of the cost function may increase during the rank reduction steps), hence we cannot immediately conclude that $f\left(X_{n_{j}}\right)-f\left(X_{n_{j}+1}\right)$ goes to zero. It does though, in view of the following argument.

Since the Riemannian optimization method (line 3 is a descent iteration (Assumption 44 and in view of line 17, we can deduce that $f\left(\tilde{X}_{n_{j}}\right)-f\left(X_{n_{j+1}}\right)>$ $c_{\mathrm{R}}\left(f\left(\tilde{X}_{n_{j}}\right)-f\left(X_{n_{j}+1}\right)\right)$ (observe the difference between $X_{n_{j+1}}$ and $\left.X_{n_{j}+1}\right)$. Besides, since $f\left(X_{n_{j}}\right)-f\left(\tilde{X}_{n_{j}}\right)>0$ (Assumption 4 ) and $c_{\mathrm{R}} \in(0,1)$, we clearly have $f\left(X_{n_{j}}\right)-f\left(\tilde{X}_{n_{j}}\right)>c_{\mathrm{R}}\left(f\left(X_{n_{j}}\right)-f\left(\tilde{X}_{n_{j}}\right)\right)$. Adding the two inequalities thus obtained, we get

$$
f\left(X_{n_{j}}\right)-f\left(X_{n_{j+1}}\right)>c_{\mathrm{R}}\left(f\left(X_{n_{j}}\right)-f\left(X_{n_{j}+1}\right)\right) .
$$

Therefore $\left\{f\left(X_{n_{j}}\right)\right\}$ is nonincreasing since $f\left(X_{n_{j}}\right)-f\left(X_{n_{j}+1}\right)>0$. Furthermore, $\left\{f\left(X_{n_{j}}\right)\right\}$ is bounded below since $\left\{X_{n}\right\}$ is bounded and $f$ is continuous (Assumption 3). Thus the sequence of differences $f\left(X_{n_{j}}\right)-f\left(X_{n_{j+1}}\right)$ must go to zero. What is more, from (7), $f\left(X_{n_{j}}\right)-f\left(X_{n_{j}+1}\right)$ must go to zero as well.

Contradiction is used to show that $\left\langle\operatorname{grad} f_{\mathrm{F}}\left(X_{n_{j}}\right), \eta_{n_{j}}^{*}\right\rangle_{X_{n_{j}}} \rightarrow 0$. Suppose not. Then there exist a subsequence $\left\{X_{n_{j}}\right\}_{j \in \mathcal{J}}$ and $\mu>0$ such that $\left\langle\operatorname{grad} f_{\mathrm{F}}\left(X_{n_{j}}\right), \eta_{n_{j}}^{*}\right\rangle_{X_{n_{j}}}<$ $-\mu$ for all $j \in \mathcal{J}$. Since $f\left(X_{n_{j}}\right)-f\left(X_{n_{j}+1}\right)$ goes to zero, it follows from (6) that $\left\{\alpha_{n_{j}}\right\}_{j \in \mathcal{J}} \rightarrow 0$. Since $\left\{\alpha_{n_{j}}\right\}_{j \in \mathcal{J}} \rightarrow 0$ and since the $\alpha_{n_{j}}$ 's are determined by Armijo backtracking (Algorithm 2), it follows that when $j$ gets sufficiently large, at least one step of backtracking is applied to get $\alpha_{n_{j}}$. Restrict the index set $\mathcal{J}$ to those (infinitely many) sufficiently large $j$ 's. Furthermore, again for all $j$ sufficiently large, in view of Definition 2 and since $\lim _{j \rightarrow \infty} X_{n_{j}}=X_{*}$, the 
safeguard (i) in the Armijo backtracking is always satisfied. Restrict further the index set $\mathcal{J}$ to those (infinitely many) sufficiently large $j$ 's. Then for all $j \in \mathcal{J}$, since $\alpha_{n_{j}}$ results from at least one step of backtracking, it must be that the update $\frac{\alpha_{n_{j}}}{\beta} \eta_{n_{j}}^{*}$ did not satisfy the Armijo condition; that is,

$$
f\left(X_{n_{j}}\right)-f\left(\tilde{R}_{X_{n_{j}}}\left(\frac{\alpha_{n_{j}}}{\beta} \eta_{n_{j}}^{*}\right)\right)<-\sigma \frac{\alpha_{n_{j}}}{\beta}\left\langle\operatorname{grad} f_{\mathrm{F}}\left(X_{n_{j}}\right), \eta_{n_{j}}^{*}\right\rangle_{X_{n_{j}}}, \quad \forall j \in \mathcal{J} .
$$

Denoting

$$
\tilde{\eta}_{n_{j}}=\frac{\eta_{n_{j}}^{*}}{\left\|\eta_{n_{j}}^{*}\right\|} \text { and } \tilde{\alpha}_{n_{j}}=\frac{\alpha_{n_{j}}\left\|\eta_{n_{j}}^{*}\right\|}{\beta}
$$

the inequality above reads

$$
\frac{\tilde{f}_{\tilde{\eta}_{n_{j}}}(0)-\tilde{f}_{\tilde{\eta}_{n_{j}}}\left(\tilde{\alpha}_{n_{j}}\right)}{\tilde{\alpha}_{n_{j}}}<-\sigma\left\langle\operatorname{grad} f_{\mathrm{F}}\left(X_{n_{j}}\right), \tilde{\eta}_{n_{j}}\right\rangle_{X_{n_{j}}}, \quad \forall j \in \mathcal{J},
$$

where $\tilde{f}_{\eta}(t):=f\left(\tilde{R}_{X}(t \eta)\right)$. If necessary, remove from $\mathcal{J}$ finitely many elements to ensure that, for all $j \in \mathcal{J}, X_{n_{j}}$ is in the neighborhood $\mathcal{U}$ of $X_{*}$ and $\tilde{\alpha}_{n_{j}}<\delta_{\mathrm{RL}}$, where $\mathcal{U}$ and $\delta_{\mathrm{RL}}$ are those of Assumption 6. The mean value theorem then ensures that, for all $j \in \mathcal{J}$, there exists $t_{n_{j}} \in\left[0, \tilde{\alpha}_{n_{j}}\right]$ such that

$$
-\left.\frac{d}{d t} \tilde{f}_{\tilde{\eta}_{n_{j}}}(t)\right|_{t=t_{n_{j}}}<-\sigma\left\langle\operatorname{grad} f_{\mathrm{F}}\left(X_{n_{j}}\right), \tilde{\eta}_{n_{j}}\right\rangle_{X_{n_{j}}}, \quad \forall j \in \mathcal{J} .
$$

Since $\left\|\eta_{n_{j}}^{*}\right\| \leq\left\|\operatorname{grad} f_{\mathrm{F}}\left(X_{n_{j}}\right)\right\|$ which remains bounded since $f \in C^{1}$ and $\left\{X_{n_{j}}\right\}$ is bounded, it follows that $\eta_{n_{j}}^{*}$ is bounded. Thus $\left\{\tilde{\alpha}_{n_{j}}\right\}_{j \in \mathcal{J}} \rightarrow 0$ since $\tilde{\alpha}_{n_{j}}=$ $\frac{\alpha_{n_{j}}\left\|\eta_{n_{j}}^{*}\right\|}{\beta}$ with $\left\{\alpha_{n_{j}}\right\}_{j \in \mathcal{J}} \rightarrow 0$. In view of property (iii) of rank-related retractions (Definition 2, we have that $\left.\frac{d}{d t} \tilde{f}_{\tilde{\eta}_{n_{j}}}(t)\right|_{t=0}=\left\langle\operatorname{grad} f_{\mathrm{F}}\left(X_{n_{j}}\right), \tilde{\eta}_{n_{j}}\right\rangle_{X_{n_{j}}}$. This and (8) yield

$$
\begin{aligned}
\left.\frac{d}{d t} \tilde{f}_{\tilde{\eta}_{n_{j}}}(t)\right|_{t=0}-\left.\frac{d}{d t} \tilde{f}_{\tilde{\eta}_{n_{j}}}(t)\right|_{t=t_{n_{j}}} & <(1-\sigma)\left\langle\operatorname{grad} f_{\mathrm{F}}\left(X_{n_{j}}\right), \tilde{\eta}_{n_{j}}\right\rangle_{X_{n_{j}}} \\
& <-(1-\sigma) \mu .
\end{aligned}
$$

We have $\left|\frac{d}{d t} \tilde{f}_{\tilde{\eta}_{n_{j}}}(t)\right|_{t=0}-\left.\frac{d}{d t} \tilde{f}_{\tilde{\eta}_{n_{j}}}(t)\right|_{t=t_{n_{j}}} \mid \leq \beta_{\mathrm{RL}} t_{n_{j}}$ in view of Assumption 6. As $j$ goes to infinity in $\mathcal{J}$, the left-hand side thus goes to zero since $t_{n_{j}} \in\left[0, \tilde{\alpha}_{n_{j}}\right]$ must go to zero. Hence $0=\lim _{j \in \mathcal{J}}\left(\left.\frac{d}{d t} \tilde{f}_{\tilde{\eta}_{n_{j}}}(t)\right|_{t=0}-\left.\frac{d}{d t} \tilde{f}_{\tilde{\eta}_{n_{j}}}(t)\right|_{t=t_{n_{j}}}\right) \leq-(1-$ $285) \mu<0$, a contradiction.

The contradiction argument is thus complete, which shows that

$$
\left\langle\operatorname{grad} f_{\mathrm{F}}\left(X_{n_{j}}\right), \eta_{n_{j}}^{*}\right\rangle_{X_{n}} \rightarrow 0
$$

In view of Lemma 1, it follows that $\eta_{n_{j}}^{*} \rightarrow 0$. We distinguish two subcases: Case 1.1: All (except finitely many) $\eta_{n_{j}}^{*}$ were obtained in line 8 with $\tilde{r}=k$. Then the claim $\lim \inf _{n \rightarrow \infty}\left\|\mathrm{P}_{\mathrm{T}_{X_{n}} \mathcal{M}_{\leq k}}\left(\operatorname{grad} f_{\mathrm{F}}\left(X_{n}\right)\right)\right\|=0$ follows. 
Case 1.2: The other case is where infinitely many $\eta_{n_{j}}^{*}$ were obtained with $\tilde{r}<k$, 290 hence with $\left\|\operatorname{grad} f_{\mathrm{F}}\left(\tilde{X}_{n_{j}}\right)-\eta_{n_{j}}^{*}\right\| \leq \epsilon_{4}\left\|\eta_{n_{j}}^{*}\right\|$ in view of line 7 . The stronger claim $\liminf \operatorname{in}_{n \rightarrow \infty}\left\|\operatorname{grad} f_{\mathrm{F}}\left(X_{n}\right)\right\|=0$ follows.

Case 2 (details): As we already pointed out, we know in Case 2 that $X_{n}$ stays in a fixed-rank manifold $\mathcal{M}_{r}$ for all $n$ large enough. Let $X_{*}$ be a limit point of $\left\{X_{n}\right\}$, and let $\left\{X_{n_{j}}\right\}$ be a subsequence that converges to $X_{*}$. Then

${ }_{295} X_{*}$ is in the closure $\overline{\mathcal{M}_{r}}$, hence in $\mathcal{M}_{\leq r}$ by Assumption 1. But $X_{*}$ cannot be in $\mathcal{M}_{\leq r-1}$, otherwise the rank reduction mechanism would not allow the rank to remain $r$. (This can be seen as follows. In Case $2, \delta_{\text {ref }}$ remains constant for all $n$ large enough. Moreover, $r \leq r_{\text {ref }}$ and $f_{\text {ref }}-f\left(\tilde{X}_{n}\right)>c_{\mathrm{R}} \delta_{\text {ref }}$ hold throughout the execution of the Algorithm 3 after the last Armijo is executed.

300 Suppose for contradiction that $X_{*} \in \mathcal{M}_{s}$ with $s<r$. Then $\tilde{X}_{n_{j}-1}$, since it is equal to $X_{n_{j}}$, gets arbitrarily close to $\mathcal{M}_{s}$. For $j$ large enough, the truncation in line 16 or 19 will produce $\hat{X}_{n_{j}-1} \in \mathcal{M}_{s} \cup \mathcal{M}_{s+1} \cdots \cup \mathcal{M}_{r-1}$ closest to $\tilde{X}_{n_{j}-1}$ and thus also arbitrarily close to $\tilde{X}_{n_{j}-1}$. Since $f$, being $C^{1}$, is uniformly continuous in any bounded domain in which the iteration stays, it follows that $\left|f\left(\hat{X}_{n_{j}-1}\right)-f\left(\tilde{X}_{n_{j}-1}\right)\right|$ becomes arbitrarily small, hence small enough for $f_{\text {ref }}-f\left(\hat{X}_{n_{j}-1}\right)>c_{\mathrm{R}} \delta_{\text {ref }}$ to hold. Hence, for some $j$ large enough, the while loop starting in line 17 will terminate with $\hat{X}_{n_{j}-1} \in \mathcal{M}_{s} \cup \mathcal{M}_{s+1} \cdots \cup \mathcal{M}_{r-1}$, meaning that the rank drops below $r$, a contradiction.) We thus conclude that $X_{*}$ is in $\mathcal{M}_{r}$. It follows from Assumption 4 that grad $f_{r}\left(X_{*}\right)=0$. We are in 310 one of two subcases:

Case 2.1: $r=k$. Then grad $f_{r}\left(X_{*}\right)=\lim _{j \rightarrow \infty} \operatorname{grad} f_{r}\left(X_{n_{j}}\right)=\lim _{j \rightarrow \infty} \mathrm{P}_{\mathrm{T}_{X_{n_{j}}} \mathcal{M}_{k}}\left(\operatorname{grad} f_{\mathrm{F}}\left(X_{n_{j}}\right)\right)=$ $\lim _{j \rightarrow \infty} \mathrm{P}_{\mathrm{T}_{X_{n_{j}}} \mathcal{M}_{\leq k}}\left(\operatorname{grad} f_{\mathrm{F}}\left(X_{n_{j}}\right)\right)$ and the claim follows.

Case 2.2: $r<k$. Then $\operatorname{grad} f_{\mathrm{F}}\left(X_{*}\right)=0$, otherwise (recall $\epsilon_{2}=0$ ) the condition in line 5 would have been satisfied and Armijo would have been exe315 cuted infinitely many times, a contradiction with Case 2. The stronger claim $\liminf _{n \rightarrow \infty}\left\|\operatorname{grad} f_{\mathrm{F}}\left(X_{n}\right)\right\|=0$ follows.

\subsection{Convergence Analysis with $\epsilon_{2} \geq 0$}

Recall that Theorem 2 considers the case where $\epsilon_{2}=0$. Its proof led us to consider Case 1 where Armijo (line 10 is executed infinitely many times.

320 Figure 2 shows a situation where this case can indeed occur. An Armijo step is taken from $X_{n} \in \mathcal{M}_{r}$ because the gradient angle condition (3) is satisfied (due to the narrowness of the valley in the cost function landscape), the next iterate is not far away since it must produce a decrease in $f$, a rank reduction to $r$ occurs at a subsequent iterate, and the process repeats over and over again. 325 This "hem stitching" phenomenon results in a slow convergence. It is tempting to remedy this phenomenon by making the iteration less inclined to leave $\mathcal{M}_{r}$.

This is the purpose of the $\epsilon_{2}$ parameter of Algorithm 3. Indeed, in the example of Figure 2, when $\epsilon_{2}>0$, the gradient distance condition (4) will finally no longer hold (because grad $f_{\mathrm{F}}\left(X_{n}\right)$ goes to zero as $n \rightarrow \infty$ ) and thus 330 line 13 will be executed instead of Armijo. The iterates thus remain on $\mathcal{M}_{r}$ and 
the Riemannian optimization method of line 3 keeps being applied, potentially offering a faster convergence.

The downside with $\epsilon_{2}>0$ is that if the minimizer $X_{*}$ of $f_{\mathrm{F}}$ is now slightly off $\mathcal{M}_{r}$ instead of being in $\mathcal{M}_{r}$, then the gradient distance condition (4) will never be satisfied, hence Armijo will never be executed, thus the rank will never increase beyond $r$, ruling out convergence to $X_{*}$. The situation, however, is not as unfavorable as it may look, much to the contrary. First, $\epsilon_{2}>0$ achieves the goal of making Algorithm 3 less inclined to increase the rank, hence obtaining a lower complexity in exchange for yielding some ground on accuracy. Second, the accuracy remains under control due to the bound on $\liminf \operatorname{in}_{n \rightarrow \infty}\left\|\mathrm{P}_{\mathrm{T}_{X_{n}} \mathcal{M}_{\leq k}}\left(\operatorname{grad} f_{\mathrm{F}}\left(X_{n}\right)\right)\right\|$ given in Theorem 3 below.

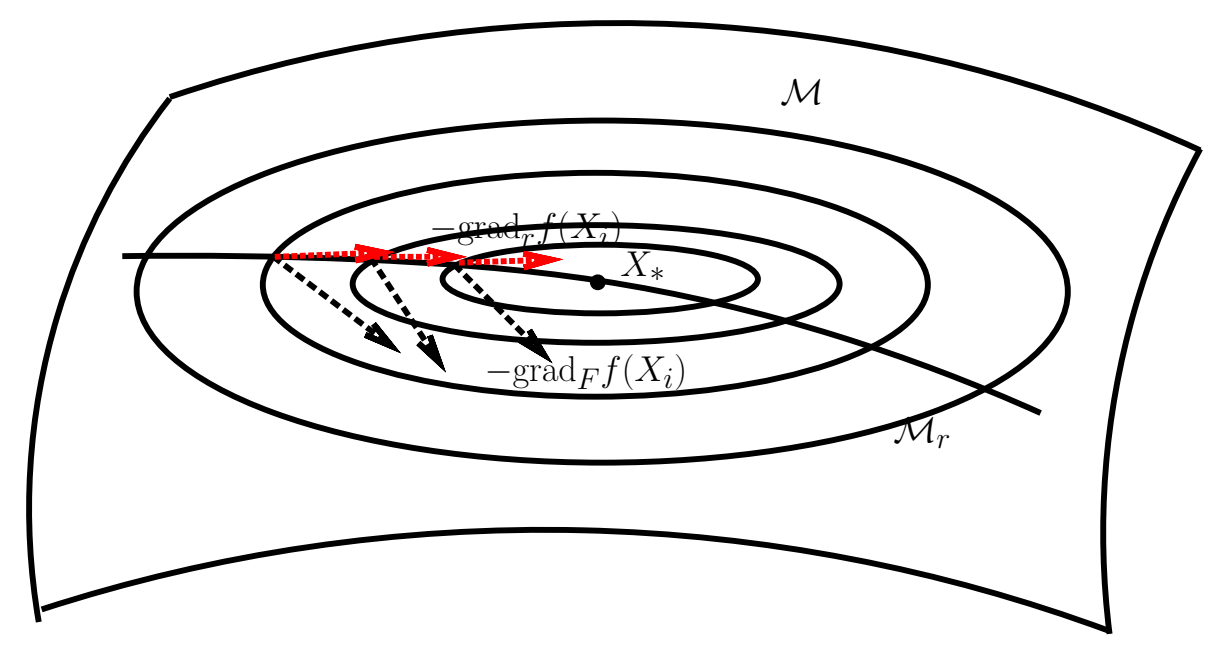

Figure 2: Illustration of a situation where the Armijo step (line 10 of Algorithm 35 would be executed infinitely many times. The black dotted arrows represent $-\operatorname{grad} f_{\mathrm{F}}$ and the red dotted arrows represent $-\operatorname{grad} f_{r}$. The circles are level sets of $f_{\mathrm{F}}$, and $X_{*}$ is a local minimum of $f_{\mathrm{F}}$ that belongs to $\mathcal{M}_{r}$.

Theorem 3. Under the standing assumptions (Section 2) and Assumptions 46. let $\left\{X_{n}\right\}$ be a bounded infinite sequence of iterates generated by Algorithm [3. now with $\epsilon_{2} \geq 0$. Then

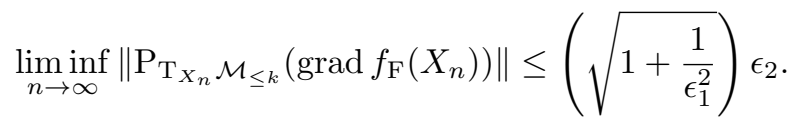

Proof. Observe that $\epsilon_{2}$ appears only in line 5 of Algorithm 3 . We will distinguish two cases, according to whether $\epsilon_{2}$ influences or not the asymptotic behavior of the iteration. To this end, let $\left\{X_{n_{j}}\right\}$ the subsequence of iterates for which the requirement in line 5 does not hold solely because the gradient distance condition (4) does not hold. In other words, we have $\left\|\operatorname{grad} f_{\mathrm{F}}\left(\tilde{X}_{n_{j}}\right)-\operatorname{grad} f_{r}\left(\tilde{X}_{n_{j}}\right)\right\|>$ 
$\epsilon_{1}\left\|\operatorname{grad} f_{r}\left(\tilde{X}_{n_{j}}\right)\right\|$ and $r<k$ but $\left\|\operatorname{grad} f_{\mathrm{F}}\left(\tilde{X}_{n_{j}}\right)-\operatorname{grad} f_{r}\left(\tilde{X}_{n_{j}}\right)\right\| \leq \epsilon_{2}$. Let $\theta$ denote the angle between $\operatorname{grad} f_{\mathrm{F}}\left(\tilde{X}_{n_{j}}\right)$ and $\operatorname{grad} f_{r}\left(\tilde{X}_{n_{j}}\right)$. We then have

$$
\sin (\theta)\left\|\operatorname{grad} f_{\mathrm{F}}\left(\tilde{X}_{n_{j}}\right)\right\|=\left\|\operatorname{grad} f_{\mathrm{F}}\left(\tilde{X}_{n_{j}}\right)-\operatorname{grad} f_{r}\left(\tilde{X}_{n_{j}}\right)\right\| \leq \epsilon_{2}
$$

and $\tan (\theta)>\epsilon_{1}$. Thus

$$
\left\|\operatorname{grad} f_{\mathrm{F}}\left(X_{n_{j}}\right)\right\| \leq \frac{1}{\sin (\theta)} \epsilon_{2}=\left(\sqrt{1+\frac{1}{\tan ^{2}(\theta)}}\right) \epsilon_{2} \leq\left(\sqrt{1+\frac{1}{\epsilon_{1}^{2}}}\right) \epsilon_{2} .
$$

Case 1: The subsequence $\left\{X_{n_{j}}\right\}$ is infinite. Then $\liminf \operatorname{in}_{n \rightarrow \infty}\left\|\operatorname{grad} f_{\mathrm{F}}\left(X_{n}\right)\right\| \leq$ $\left(\sqrt{1+\frac{1}{\epsilon_{1}^{2}}}\right) \epsilon_{2}$, and the conclusion follows.

Case 2: The subsequence $\left\{X_{n_{j}}\right\}$ is finite. Let $X_{K}$ be its last element. Then the exact same sequence $\left\{X_{n}\right\}$ would be generated by setting $\epsilon_{2}$ to zero from iteration $K+1$ onward. One can then conclude as in Theorem 2 that $\liminf \operatorname{in}_{n \rightarrow \infty}\left\|\mathrm{P}_{\mathrm{T}_{X_{n}} \mathcal{M}_{\leq k}}\left(\operatorname{grad} f_{\mathrm{F}}\left(X_{n}\right)\right)\right\|=0$.

\section{Implementation Details}

A practical implementation of Algorithm 3 requires an adequate way to store the low-rank iterates $X_{n}$ and the various tangent vectors, to compute the projection required in line 8 , and to choose the rank-related retraction $\tilde{R}$ required in line 10. We present those implementation details for the frequently encountered case where

$$
\mathcal{M}=\mathbb{R}^{m \times n} .
$$

Let $X \in \mathbb{R}_{r}^{m \times n}$. Then $X$ can be decomposed as $X=U_{r} D_{r} V_{r}^{T}$ where $U_{r}$ and $V_{r}$ are orthonormal matrices of size $m \times r$ and $n \times r$ respectively. We have (see [21, Theorem 3.2]):

$$
\mathrm{T}_{X} \mathbb{R}_{\leq \tilde{r}}^{m \times n}=\left\{\begin{array}{r}
U_{r} A V_{r}^{T}+U_{r} B V_{r \perp}^{T}+U_{r \perp} C V_{r}^{T}+U_{r \perp} E V_{r \perp}^{T}: \\
A, B, C, E \text { arbitrary matrices with } \operatorname{rank}(E) \leq \tilde{r}-r,
\end{array}\right\},
$$

where $U_{r \perp}$ is chosen such that $\left[\begin{array}{ll}U_{r} & U_{r \perp}\end{array}\right]$ is an orthogonal matrix, and likewise for $V_{r \perp}$. (An expression for the case where $\mathcal{M}$ is the Frobenius sphere can also be found in 28.)

Thus the elements $\eta$ of $\mathrm{T}_{X} \mathbb{R}_{\leq \tilde{r}}^{m \times n}$ are the matrices of the form

$$
\eta=\left[\begin{array}{ll}
U_{r} & U_{r \perp}
\end{array}\right]\left[\begin{array}{ll}
A & B \\
C & E
\end{array}\right]\left[\begin{array}{c}
V_{r}^{T} \\
V_{r \perp}^{T}
\end{array}\right], \quad \operatorname{rank}(E) \leq \tilde{r}-r .
$$

Since $E$ has rank at most $\tilde{r}-r=: \Delta r$, we can write $E=\left[\begin{array}{ll}\tilde{U}_{\Delta r} & \tilde{U}_{\Delta r \perp}\end{array}\right]\left[\begin{array}{cc}E_{\Delta r} & 0 \\ 0 & 0\end{array}\right]\left[\begin{array}{cc}\tilde{V}_{\Delta r} & \tilde{V}_{\Delta r \perp}\end{array}\right]^{T}$ 
with $E_{\Delta r}$ of size $\Delta r \times \Delta r$, and $(9)$ becomes

$$
\begin{aligned}
\eta & =\left[\begin{array}{lll}
U_{r} & U_{\Delta r} & U_{(r+\Delta r) \perp}
\end{array}\right]\left[\begin{array}{ccc}
A & B_{1} & B_{2} \\
C_{1} & E_{\Delta r} & 0_{\Delta r \times(n-\tilde{r})} \\
C_{2} & 0_{(m-\tilde{r}) \times \Delta r} & 0_{(m-\tilde{r}) \times(n-\tilde{r})}
\end{array}\right]\left[\begin{array}{c}
V_{r}^{T} \\
V_{\Delta r}^{T} \\
V_{(r+\Delta r) \perp}^{T}
\end{array}\right] \\
& =\left[\begin{array}{ll}
U_{\tilde{r}} & U_{\tilde{r} \perp}
\end{array}\right]\left[\begin{array}{cc}
\dot{D}_{\tilde{r}} & B_{2} \\
C_{2} & 0_{(m-\tilde{r}) \times(n-\tilde{r})}
\end{array}\right]\left[\begin{array}{c}
V_{\tilde{r}}^{T} \\
V_{\tilde{r} \perp}^{T}
\end{array}\right]
\end{aligned}
$$

with $U_{\Delta r}=U_{r \perp} \tilde{U}_{\Delta r}, U_{(r+\Delta t) \perp}=U_{r \perp} \tilde{U}_{\Delta r \perp}$ and likewise for $V$. Since $X=$ $U_{r} D_{r} V_{r}^{T}$, it can be written in the same block structure, yielding

$$
\begin{aligned}
X & =\left[\begin{array}{lll}
U_{r} & U_{\Delta r} & U_{(r+\Delta r) \perp}
\end{array}\right]\left[\begin{array}{ccc}
D_{r} & 0_{r \times \Delta r} & 0_{r \times(n-\tilde{r})} \\
0_{\Delta r \times r} & 0_{\Delta r \times \Delta r} & 0_{\Delta r \times(n-\tilde{r})} \\
0_{(m-\tilde{r}) \times r} & 0_{(m-\tilde{r}) \times \Delta r} & 0_{(m-\tilde{r}) \times(n-\tilde{r})}
\end{array}\right]\left[\begin{array}{c}
V_{r}^{T} \\
V_{\Delta r}^{T} \\
V_{(r+\Delta r) \perp}^{T}
\end{array}\right] \\
& =\left[\begin{array}{ll}
U_{\tilde{r}} & U_{\tilde{r} \perp}
\end{array}\right]\left[\begin{array}{cc}
D_{\tilde{r}} & 0_{\tilde{r} \times(n-\tilde{r})} \\
0_{(m-\tilde{r}) \times \tilde{r}} & 0_{(m-\tilde{r}) \times(n-\tilde{r})}
\end{array}\right]\left[\begin{array}{c}
V_{\tilde{r}}^{T} \\
V_{\tilde{r} \perp}^{T}
\end{array}\right] .
\end{aligned}
$$

Expression 10 can be rewritten as

$$
\eta=\dot{U}_{\tilde{r}} D_{\tilde{r}} V_{\tilde{r}}^{T}+U_{\tilde{r}} \dot{D}_{\tilde{r}} V_{\tilde{r}}^{T}+U_{\tilde{r}} D_{\tilde{r}} \dot{V}_{\tilde{r}}^{T},
$$

with $U_{\tilde{r}}^{T} \dot{U}_{\tilde{r}}=0, V_{\tilde{r}}^{T} \dot{V}_{\tilde{r}}=0$, imposing that the last $\Delta r$ columns of $\dot{U}_{\tilde{r}}$ and $\dot{V}_{\tilde{r}}$ are zero, and imposing that the first $r$ columns of $U_{\tilde{r}}$ (resp. $\left.V_{\tilde{r}}\right)$ are $U_{r}$ (resp. $V_{r}$ ).

The tangent space to $\mathbb{R}^{m \times n}$ is a copy of $\mathbb{R}^{m \times n}$. Let $\xi \in \mathbb{R}^{m \times n}$. Then $\xi$ admits a unique decomposition

$$
\xi=\left[\begin{array}{ll}
U_{r} & U_{r \perp}
\end{array}\right]\left[\begin{array}{ll}
A & B \\
C & E
\end{array}\right]\left[\begin{array}{c}
V_{r}^{T} \\
V_{r \perp}^{T}
\end{array}\right],
$$

and $\eta^{*} \in \arg \min _{\eta \in \mathrm{T}_{X} \mathcal{M}_{\leq \tilde{r}}}\|\xi-\eta\|$ if and only if

$$
\eta=\left[\begin{array}{ll}
U_{r} & U_{r \perp}
\end{array}\right]\left[\begin{array}{ll}
A & B \\
C & \tilde{E}
\end{array}\right]\left[\begin{array}{c}
V_{r}^{T} \\
V_{r \perp}^{T}
\end{array}\right]
$$

where $\tilde{E}$ is a best (in the Frobenius norm) rank- $(\tilde{r}-r)$ approximation of $E$; see [21, Corollary 3.3].

One possible choice for the rank-related retraction (Definition 2 ) is the following one, which relies on the decomposition (11):

$$
\tilde{R}_{X}(\eta)=\tilde{U}_{+} \tilde{D}_{+} \tilde{V}_{+},
$$

where

$$
\begin{aligned}
& \tilde{U}_{+}=\left(U_{\tilde{r}}+\dot{U}_{\tilde{r}}\right) S_{U}^{-1}, \\
& \tilde{V}_{+}=\left(V_{\tilde{r}}+\dot{V}_{\tilde{r}}\right) S_{V}^{-1}, \\
& \tilde{D}_{+}=S_{U}\left(D_{\tilde{r}}+\dot{D}_{\tilde{r}}\right) S_{V}^{T},
\end{aligned}
$$

where $S_{U}$ and $S_{V}$ can be chosen freely, e.g., to orthonormalize the factors $\tilde{U}_{+}$ and $\tilde{V}_{+}$. This rank-related retraction reduces to the RRR retraction of [29, $\left.\S 3.4\right]$ when $\eta \in \mathrm{T}_{X} \mathbb{R}_{r}^{m \times n}$. Other rank-related retractions are given in [23, §4.3.5]. 


\section{Application}

In order to illustrate the potential of the rank-adaptive mechanism introduced in Algorithm 3, we present numerical experiments for the well-known weighted low-rank approximation problem:

$$
\min _{X \in \mathcal{M}_{\leq k}}\|A-X\|_{W}^{2}, \quad f(X)=\|A-X\|_{W}^{2}=\operatorname{vec}\{A-X\}^{T} W \operatorname{vec}\{A-X\}
$$

where $\mathcal{M}=\mathbb{R}^{m \times n}, A$ is given, $W \in \mathbb{R}^{m n \times m n}$ is a positive definite symmetric weighting matrix, and vec $\{A\}$ denotes the vectorized form of $A$, i.e., a vector constructed by stacking the consecutive columns of $A$ in one vector. This problem has several applications, notably in machine learning [30].

In our experiments, the matrix $A$ is generated as $A_{1} A_{2}^{T} \in \mathbb{R}^{m \times n}$, where $A_{1} \in \mathbb{R}^{m \times r}$ and $A_{2} \in \mathbb{R}^{n \times r}$ are drawn from the standard Gaussian distribution. The weighting matrix $W$ is generated as $W=U \Sigma U^{T}$, where $U$ is obtained by orthonormalizing a matrix drawn from the standard Gaussian distribution and $\Sigma$ is a diagonal matrix whose diagonal is a vector of logarithmically spaced 370 points between $10^{-2}$ and 1 multiplied element-wise by a vector drawn from the uniform distribution on $[0.5,1.5]$. We take $m=100, n=15, r=5$. Three values are considered for the rank bound $k$, one less than the true rank $r$, one equal to the true rank, and one greater than the true rank.

Four algorithms are compared: DMM 31, SULS 21, APM 32, and RRAM (Algorithm 3). We use the publicly available Matlab implementation of APM ${ }^{2}$ and our own implementation of the other algorithms ${ }^{3}$ In RRAM, the Riemannian update (line 3 of Algorithm 3) is performed by means of the same Riemannian steepest-descent scheme as in SULS.

The initial iterate of RRAM and SULS is taken as $U_{0} \Sigma_{0} V_{0}^{T}$, where $U_{0}$, resp. $V_{0}$, is obtained by orthonormalizing a matrix of size $m \times k$, resp. $n \times k$, drawn from the standard Gaussian distribution, and $\Sigma_{0}$ is a diagonal matrix with entries drawn from the uniform distribution on $[0,1]$. The initial points of DMM and APM are randomly generated $n$-by- $(n-k)$ and $m$-by- $(m-k)$ matrices respectively. RRAM (Algorithm 3 ) is run with $\epsilon_{1}=\sqrt{3}, \epsilon_{2}=10^{-4}$, ${ }_{385} \epsilon_{4}=\frac{\epsilon_{1}}{2}, \Delta_{0}=10^{-2}$.

The results shown in Table 1 are the average of 10 runs for different data matrices $A$, weighting matrices $W$, and initial points. All the results are obtained with Matlab version 8.3.0 (R2014a) for Linux on a platform with Intel(R) Core(TM) i7-4770 CPU at $3.4 \mathrm{GHz}$ with 16GB memory.

390 For $k=3<r$ and $k=5=r$, the rank of the iterates of RRAM remains equal to $k$, hence it performs very similarly to SULS. Observe that these two algorithms clearly outperform the two others.

For $k=10>r$, the rank update mechanism of RRAM reduces the rank of the iterates from $k$ to $r$. In view of the chosen stopping criterion, SULS

\footnotetext{
2 ftp://ftp.esat.kuleuven.be/sista/markovsky/abstracts/04-220.html

3 http://sites.uclouvain.be/absil/2015.05
} 


\begin{tabular}{cccccc}
\hline $\mathrm{k}$ & method & rank & $\mathrm{f}$ & Relative Error & time(sec) \\
\hline $\mathrm{k}=3$ & RRAM & 3.0 & $1.833_{+02}$ & $3.410_{-01}$ & $9.271_{-01}$ \\
& SULS & 3.0 & $1.833_{+02}$ & $3.410_{-01}$ & $9.161_{-01}$ \\
& DMM & 3.0 & $1.833_{+02}$ & $3.410_{-01}$ & $2.566_{+00}$ \\
& APM & 3.0 & $1.822_{+02}$ & $3.401_{-01}$ & $1.836_{+00}$ \\
\hline $\mathrm{k}=5$ & RRAM & 5.0 & $6.752_{-12}$ & $6.751_{-08}$ & $3.665_{-01}$ \\
& SULS & 5.0 & $6.752_{-12}$ & $6.751_{-08}$ & $3.507_{-01}$ \\
& DMM & 5.0 & $4.432_{-11}$ & $9.460_{-08}$ & $2.262_{+00}$ \\
& APM & 5.0 & $3.740_{-09}$ & $1.439_{-06}$ & $1.166_{+00}$ \\
\hline $\mathrm{k}=10$ & RRAM & $5.0(10 / 10)$ & $6.434_{-12}$ & $6.345_{-08}$ & $4.733_{-01}$ \\
& SULS & $10.0(0 / 10)$ & $9.483_{-12}$ & $7.704_{-08}$ & $9.264_{-01}$ \\
& DMM & $10.0(0 / 10)$ & $3.798_{-11}$ & $8.657_{-08}$ & $1.034_{+00}$ \\
& APM & $10.0(0 / 10)$ & $9.623_{-09}$ & $2.270_{-06}$ & $2.946_{+00}$ \\
\hline
\end{tabular}

Table 1: Method comparisons. The number in the parenthesis indicates the fraction of experiments where the numerical rank (number of singular values greater than $10^{-8}$ ) found by the algorithm equals the true rank. The subscript $\pm n$ indicates a scale of $10^{ \pm n}$. RRAM (Algorithm 3) and SULS are stopped when the norm of the final gradient on the fixed-rank manifold over the norm of initial full gradient is less than $10^{-7}$ while DMM and APM are stopped when the norm of final gradient over the norm of initial gradient is less than $10^{-7}$.

and RRAM terminate with a comparable accuracy, but RRAM is considerably faster. The effect of the rank-adaptive mechanism as the iteration proceeds is visible on Figure 3.

\section{Conclusions and perspectives}

In this paper, we have proposed a new algorithm (Algorithm 3 ) for minimizing a real-valued function on a manifold $\mathcal{M}$ under an additional rank inequality constraint. Rank update mechanisms, based on certain rank-related objects, have been defined to facilitate efficiently finding a suitable rank. Instances of those objects have been provided for the case where $\mathcal{M}$ is the matrix space $\mathbb{R}^{m \times n}$. They can readily be adapted to the case where $\mathcal{M}$ is the Frobenius

405 sphere, and the way is open for handling other cases. The convergence properties of the algorithm have been analyzed, and it has been shown to outperform state-of-the-art methods on a weighted low-rank approximation problem.

This work opens several avenues for further research, e.g., to improve the efficiency of Algorithm 3, set its parameters, strengthen its convergence analysis, investigate the use of other Riemannian optimization algorithms for the Riemannian update (line 3), and test the method on other low-rank optimization problems.

\section{References}

[1] B. Vandereycken, Low-rank matrix completion by Riemannian optimization, SIAM Journal on Optimization 23 (2) (2013) 1214-1236. 


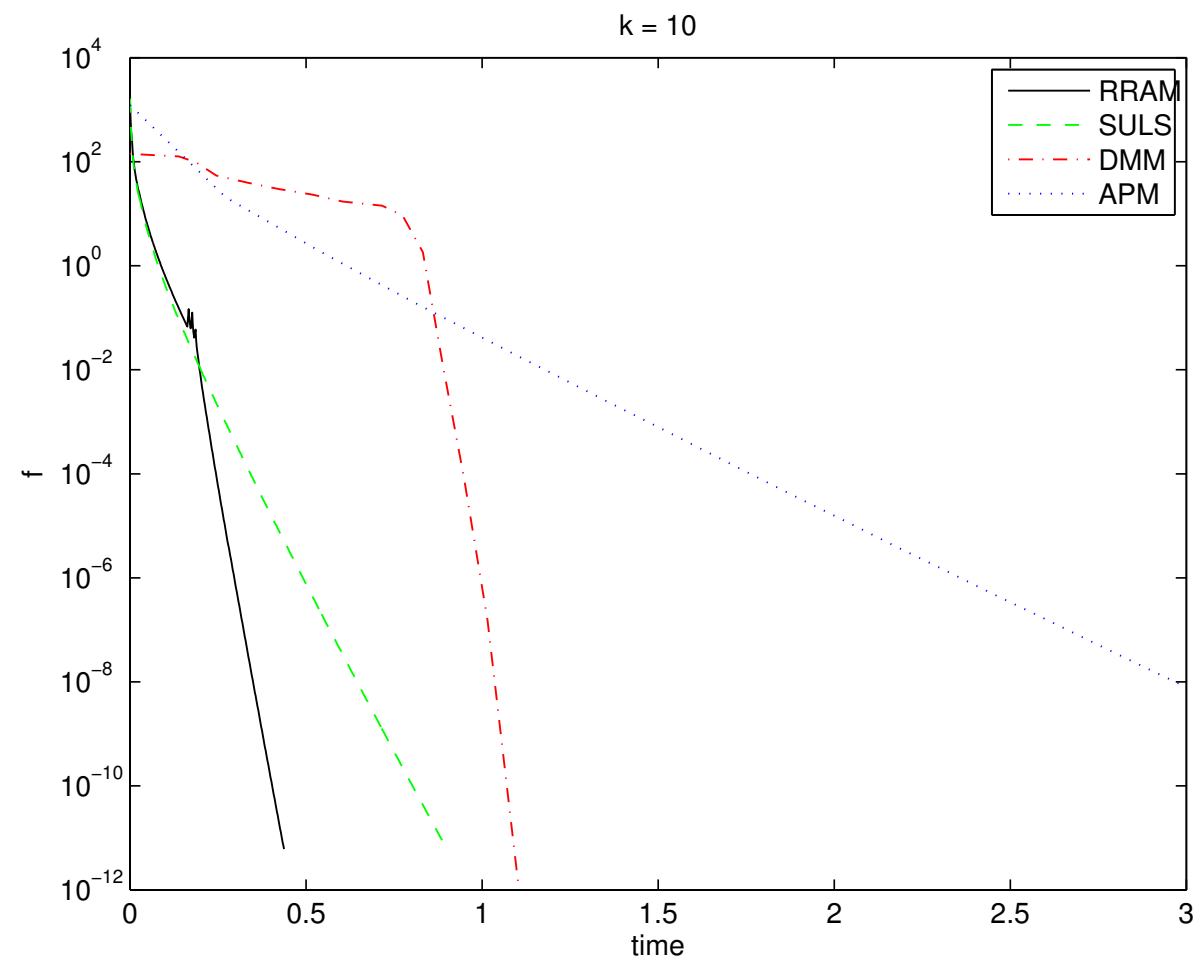

Figure 3: Objective function value against computational time with $k$ is greater than true rank.

n [2] L. Cambier, P.-A. Absil, Robust low-rank matrix completion by Riemannian optimization, Tech. Rep. UCL-INMA-2015.04, UCLouvain (2015). URL http://sites.uclouvain.be/absil/2015.04

[3] Y. Amit, M. Fink, N. Srebro, S. Ullman, Uncovering shared structures in multiclass classification, in: Proceedings of the 24th International Conference on Machine Learning, ICML '07, ACM, New York, NY, USA, 2007, pp. 17-24. doi:10.1145/1273496.1273499.

URL http://doi .acm.org/10.1145/1273496.1273499

[4] B. Mishra, G. Meyer, F. Bach, R. Sepulchre, Low-rank optimization with

425 trace norm penalty, SIAM Journal on Optimization 23 (4) (2013) 2124-

n 2149. arXiv:http://dx.doi.org/10.1137/110859646 doi:10.1137/ 110859646 .

URL http://dx.doi.org/10.1137/110859646

[5] Q. Yan, J. Ye, X. Shen, Simultaneous pursuit of sparseness and rank structures for matrix decomposition, Journal of Machine Learning Research 16 
(2015) 47-75.

URL http://jmlr.org/papers/v16/yan15a.html

[6] J. Abernethy, F. Bach, T. Evgeniou, J.-P. Vert, A new approach to collaborative filtering: Operator estimation with spectral regularization, Journal of Machine Learning Research 10 (2009) 803-826. URL http://www.jmlr.org/papers/v10/abernethy09a.html

n [7] U. Shalit, D. Weinshall, G. Chechik, Online learning in the embedded manifold of low-rank matrices, Journal of Machine Learning Research 13 (1) (2012) 429-458. URL http://dl .acm.org/citation. cfm?id=2503308.2188399

[8] I. Markovsky, Low Rank Approximation: Algorithms, Implementation, Applications, Springer, 2012. doi:10.1007/978-1-4471-2227-2. URL http://homepages.vub.ac.be/ imarkovs/book.html

[9] M. Fazel, T. K. Pong, D. Sun, P. Tseng, Hankel matrix rank minimization with applications to system identification and realization, SIAM Journal on Matrix Analysis and Applications 34 (3) (2013) 946-977. doi:10.1137/ 110853996.

[10] G. Liu, Z. Lin, S. Yan, J. Sun, Y. Yu, Y. Ma, Robust recovery of subspace structures by low-rank representation, IEEE Transactions on Pattern Analysis and Machine Intelligence 35 (1) (2013) 171-184. doi: 10.1109/TPAMI.2012.88.

[11] F. Seidel, C. Hage, M. Kleinsteuber, pROST: a smoothed lp-norm robust online subspace tracking method for background subtraction in video, Machine Vision and Applications 25 (5) (2013) 1227-1240. doi:10.1007/ s00138-013-0555-4.

[12] B. Mishra, G. Meyer, F. Bach, R. Sepulchre, Low-rank optimization with trace norm penalty, SIAM Journal on Optimization 23 (4) (2013) 21242149. doi:10.1137/110859646.

[13] P.-A. Absil, L. Amodei, G. Meyer, Two Newton methods on the manifold of fixed-rank matrices endowed with Riemannian quotient geometries, Computational Statistics 29 (3-4) (2014) 569-590. doi:10.1007/ s00180-013-0441-6.

[14] B. Mishra, G. Meyer, S. Bonnabel, R. Sepulchre, Fixed-rank matrix factorizations and Riemannian low-rank optimization, Computational Statistics 29 (3-4) (2014) 591-621. doi:10.1007/s00180-013-0464-z URL http://dx.doi.org/10.1007/s00180-013-0464-z

[15] U. Helmke, J. B. Moore, Optimization and Dynamical Systems, Communications and Control Engineering Series, Springer-Verlag London Ltd., London, 1994, with a foreword by R. Brockett. 
[16] P.-A. Absil, R. Mahony, R. Sepulchre, Optimization Algorithms on Matrix Manifolds, Princeton University Press, Princeton, NJ, 2008.

[17] W. Ring, B. Wirth, Optimization methods on Riemannian manifolds and their application to shape space, SIAM Journal on Optimization 22 (2) (2012) 596-627. doi:10.1137/11082885X

[18] W. Huang, P.-A. Absil, K. A. Gallivan, A Riemannian symmetric rank-one trust-region method, Mathematical Programming, Series A 150 (2) (2014) 179-216. doi:10.1007/s10107-014-0765-1 URL http://sites.uclouvain.be/absil/2013.03

[19] W. Huang, K. A. Gallivan, P.-A. Absil, A Broyden class of quasi-Newton methods for Riemannian optimization, SIAM Journal on Optimization 25 (3) (2015) 1660-1685. doi:10.1137/140955483. URL http://sites.uclouvain.be/absil/2014.01

[20] H. Sato, A Dai-Yuan-type Riemannian conjugate gradient method with the weak Wolfe conditions (2014). arXiv:1405.4371.

[21] R. Schneider, A. Uschmajew, Convergence results for projected line-search methods on varieties of low-rank matrices via Łojasiewicz inequality, SIAM

1) Journal on Optimization 25 (1) (2015) 622-646. arXiv:1402.5284, doi: $10.1137 / 140957822$

[22] A. Uschmajew, B. Vandereycken, Line-search methods and rank increase on low-rank matrix varieties, in: Proceedings of the 2014 International Symposium on Nonlinear Theory and its Applications (NOLTA2014), 2014.

[23] G. Zhou, Rank-contrained optimization: A Riemannian manifold approach, Ph.D. thesis, Florida State University (2015).

[24] R. L. Adler, J.-P. Dedieu, J. Y. Margulies, M. Martens, M. Shub, Newton's method on Riemannian manifolds and a geometric model for the human spine, IMA Journal of Numerical Analysis 22 (3) (2002) 359-390.

[25] N. Boumal, B. Mishra, P.-A. Absil, R. Sepulchre, Manopt, a Matlab toolbox for optimization on manifolds, Journal of Machine Learning Research 15 (2014) 1455-1459.

$500 \quad$ URL http://jmlr.org/papers/v15/boumal14a.html

[26] J. Nocedal, S. Wright, Numerical optimization, 2nd Edition, Springer series in operations research and financial engineering, Springer, New York, NY, 2006.

[27] P.-A. Absil, K. A. Gallivan, Accelerated line-search and trust-region methods, SIAM Journal on Numerical Analysis 47 (2) (2009) 997-1018. 
[28] T. P. Cason, P.-A. Absil, P. Van Dooren, Iterative methods for low rank approximation of graph similarity matrices, Linear Algebra and its Applications 438 (4) (2013) 1863 - 1882, 16th ILAS Conference Proceedings, Pisa 2010. doi:10.1016/j.laa.2011.12.004

510

[32] W.-S. Lu, S.-C. Pei, P.-H. Wang, Weighted low-rank approximation of general complex matrices and its application in the design of 2-d digital filters, IEEE Transactions on Circuits and Systems I 44 (1997) 650-655. 